\title{
Enfermedades prevalentes de la infancia detectadas en atención odontológica y prácticas clave AIEPI
}

\section{Prevalent childhood illnesses detected in dental care and IMCI key practices}

\author{
Shyrley Díaz-Cardenas (D)', Sthefanie del Carmen Perez-Puello (D)², Ketty del Rosario Ramos-Martínez (iD) \\ 1. Universidad de Cartagena. Cartagena, Colombia. Correo: sdiazc@unicartagena.edu.co - https://orcid.org/0000-0003-1967-8981 \\ 2. Corporación Universitaria Rafael Núñez. Cartagena. Colombia. Correo: sthefanie.perez@curnvirtual.edu.co - https://orcid.org/0000-0001-9145-8217 \\ 3. Universidad de Cartagena. Colombia. Correo: kramos1@unicartagena.edu.co - https://orcid.org/0000-0001-9123-8636
}

Tipología: Artículo de investigación científica y tecnológica

Para citar este artículo: Díaz-Cardenas S, Perez-Puello S, Ramos-Martínez K. Enfermedades prevalentes de la infancia detectadas en atención odontológica y prácticas clave AIEPI. Duazary. 2021 enero; 18(1): 57-70. Doi: https://doi.org/10.21676/2389783X.3821

Palabras

clave:

Atención Integrada a las Enfermedades Prevalentes de la Infancia; prevalencia; salud del niño; medicina familiar y comunitaria; atención odontológica.

Keywords: Integrated M anagement $\mathrm{O}$ f Childhood II Iness; Prevalence; Child Health; Family Practice; Dental Care.

\section{RESUMEN}

En el presente estudio se asociaron Enfermedades Prevalentes en la Infancia (EPI), detectadas en atención odontológica, con variables sociodemográficas y prácticas claves AIEPI (Atención Integral a Enfermedades Prevalentes de la Infancia) en binomios madres-niños, menores de 5 años, afrodescendientes de Cartagena-Colombia. Se realizó un estudio descriptivo transversal en madres de 23.400 niños menores de 5 años. La muestra fue de 548 binomios seleccionados por muestreo probabilístico aleatorio durante atención odontológica en Jornada de Salud. Se aplicó instrumento de investigaciones operativas en AIEPI (cuestionario); se estimaron EPI ocurridas en los últimos 7 días y prácticas claves AIEPI realizadas frente a estas. Se reportaron frecuencias, proporciones, regresión de Poisson con varianza robusta para asociar EPI con variables sociodemográficas y prácticas claves AIEPI. Las EPI detectadas desde la atención odontológica fueron principalmente resfriado, caries dental y diarrea; la práctica clave AIEPI más realizada fue lactancia materna exclusiva $(80,4 \%)$. Hubo asociación entre la aparición de EPI y no contar con servicios de salud público ( $R P=1,16 ; p=0,023)$, no cepillar los dientes de los niños después de tomar biberón o mamar y antes de dormir $(R P=1,08 ; p=0,022$ y $R P=0,90$; $\mathrm{p}=0,006$ respectivamente). Desde la atención odontológica pueden ser detectadas EPI para su tratamiento oportuno y realizar consejería preventiva atendiendo de forma integral a la primera infancia.

\section{ABSTRACT}

In the present study, Childhood Illnesses ( $\mathrm{Cl}$ ) detected in dental care were associated with sociodemographic variables and IMCI (Integrated Management for Childhood Illness) key practices. It was carried out in mother-child binomials, under five years old, afro descendants from CartagenaColombia. A descriptive cross-sectional study was conducted on mothers of 23,400 children under five years. The sample was 548 binomials, selected by random probability sampling during dental care at Jornada de Salud. An operational research instrument was applied to $\mathrm{IMCl}$ (questionnaire); $\mathrm{Cl}$ that occurred in the last seven days and $\mathrm{IMCl}$ key practices performed against them were estimated. Frequencies, proportions, Poisson regression with robust variance were reported to associate $\mathrm{Cl}$ with sociodemographic variables, and IMCI key practices. The $\mathrm{Cl}$ detected from dental care were mainly colds, dental caries, and diarrhea; the most performed IMCI key practice was exclusive breastfeeding (80.4\%). There was an association between the $\mathrm{Cl}$ and no public health services ( $\mathrm{RP}=1.16 ; \mathrm{p}=0.023)$, not brushing children's teeth after bottle or breastfeeding and before bed $(R P=1.08 ; p=0.022$ and $R P=0.90 ; p=$ 
0.006, respectively). From dental care, $\mathrm{Cl}$ can be detected for its timely treatment and perform preventive counseling attending to early childhood comprehensively.

\section{INTRODUCCIÓN}

En la actualidad, las altas prevalencias de enfermedades en la primera infancia han generado la necesidad de implementar estrategias, intervenciones y políticas públicas que permitan mejorar el cuidado, la prevención y la disminución de las tasas de mortalidad y morbilidad en los grupos poblacionales vulnerables ${ }^{1}$. Dentro de las enfermedades más prevalentes en la primera infancia se encuentran: insuficiencia respiratoria aguda (IRA), resfriado, bronquitis, neumonía, diarrea, dengue, inmunoprevenibles, caries dental, otitis, desnutrición, entre otras ${ }^{2}$.

La Organización Mundial de la Salud (OMS) reportó 5,9 millones de muertes de niños menores de 5 años en el 2015, evidenciando una disminución en las cifras $^{3}$ al ser comparadas con las reportadas para el año 1990, de 12,4 millones de muertes por año ${ }^{4}$. A pesar de este descenso, las cifras continúan siendo alarmantes y se alejan de los objetivos del Desarrollo Sostenible en relación con prevención de la mortalidad infantil, reportando que, para el 2030, en 63 países la tasa de mortalidad infantil deberá ser de 12 muertes por cada 1000 nacidos vivos ${ }^{3}$.

Dentro de los factores relacionados con la presencia de estas enfermedades en la infancia se encuentra edad de la madre ${ }^{5}$, nivel de escolaridad de cuidadores $^{5}$, tipo de servicio de salud ${ }^{6}$ y prácticas de cuidado en salud general ${ }^{6}$ y bucal ${ }^{7}$, entre otros. En consecuencia, la OMS y el Fondo de las Naciones Unidas para la Infancia (UNICEF) han desarrollado la estrategia Atención Integral a Enfermedades de la Primera Infancia (AIEPI), con el objetivo de disminuir las tasas de mortalidad infantil, las enfermedades y promover cuidados que contribuyan al desarrollo de los niños menores de 5 años $^{8}$. La estrategia permite identificar señales de riesgo que podrían poner en peligro la vida del infante ${ }^{9}$, siendo efectiva en comunidades vulnerables que experimentan inequidades en salud y barreras económicas, sociales y culturales que podrían ser determinantes en el proceso-salud enfermedad de los infantes ${ }^{10} y$ en las prácticas de los cuidadores.
La formación de profesionales de la salud en el abordaje de casos utilizando los principios de la estrategia AIEPI, el mejoramiento de los sistemas de salud y las prácticas implementadas por los cuidadores en el hogar también son componentes del enfoque integral de la estrategia ${ }^{11}$. Esta formación es fundamental, considerando la ausencia y la inequidad vivenciada en algunos centros asistenciales de salud al no brindarse una atención integral a través de programas como inmunización, crecimiento y desarrollo, y evaluación y seguimiento de la alimentación, lo que favorece el incremento de las tasas de morbilidad y mortalidad infantil ${ }^{12}$. Por lo tanto, es importante el reconocimiento temprano de factores de riesgo y tratamiento de las enfermedades por parte de los profesionales de la salud ${ }^{12}$. El entrenamiento de profesionales de la salud en la estrategia AIEPI mejora sus habilidades con respecto a la clasificación de las enfermedades, la prescripción de medicamentos, la vacunación de los niños y la consejería sobre prácticas clave como la nutrición ${ }^{11}$.

En algunos estudios ${ }^{13-16}$ no se hallan reportes sobre el papel del odontólogo dentro del equipo interdisciplinar inmerso en la implementación de la estrategia AIEPI. Sin embargo, los profesionales de la salud bucal también representan un recurso de apoyo importante desde la atención odontológica a la primera infancia al realizar un abordaje integral. Consecuentemente, no solo debe primar el motivo de consulta, que para su caso frecuentemente corresponde a caries dental o trauma dentoalveolar, enfermedades bucales prevalentes en la infancia, sino que también es relevante identificar signos y síntomas de riesgo de las demás enfermedades prevalentes de la infancia según AIEPI. Lo anterior con el objetivo de remitir al paciente para su manejo y tratamiento oportuno y participar en la consejería de algunos aspectos importantes para el desarrollo del niño y la familia, como la lactancia materna y la alimentación complementaria, cuyos beneficios también influyen en el desarrollo del aparato estomatognático, la oclusión y la prevención de la caries dental ${ }^{17}$. Además, se optimizaría cada 
contacto en la consulta para realizar consejería a la madre sobre nutrición, según indica la estrategia AEIPI, lo cual, de acuerdo con un estudio realizado en profesionales de la salud, podría mejorar su comunicación con el cuidador ${ }^{18}$.

Teniendo en cuenta que en Cartagena se reporta una alta prevalencia de diarrea en un $71,7 \%{ }^{17}$, resfriado común de $99,2 \%{ }^{17}$, otitis del $2,15 \%{ }^{17}$ y caries dental del $51 \%{ }^{19}$ en niños menores de 5 años, y en general la carga de morbilidad y mortalidad observada en Colombia para esta población, la Facultad de Odontología de la Universidad de Cartagena, Colombia, posee 10 años de experiencia en el entrenamiento sobre la estrategia AIEPI a estudiantes de Odontología, Odontólogos y Odontopediatras. Actualmente no se dispone de evidencias en la literatura que reporten la detección temprana de EPI en el marco de la estrategia AIEPI desde la consulta odontológica para su remisión y posterior intervención oportuna. Por lo tanto, el objetivo del estudio fue reportar la prevalencia de EPI detectadas desde la atención odontológica y asociarlas con variables sociodemográficas y prácticas claves AIEPI reportadas por madres de niños afrodescendientes de la Ciudad de Cartagena de Indias-Colombia. Todo con el fin de conocer el comportamiento epidemiológico de la primera infancia que acude a los servicios de salud bucal y justificar la importancia en la integralidad de la atención desde la odontología, a través de la implementación de la estrategia AIEPI.

\section{MATERIALES Y MÉTODOS}

\section{Tipo de estudio y muestra}

Estudio analítico de corte transversal realizado en una población marco de 23.400 madres de niños menores de 5 años, calculando una muestra de 548 binomios madres-niños menores de 5 años afrodescendientes, pertenecientes a comunidades costeras de Cartagena (Boquilla, Tierra Baja y Caño del Oro) 2014-2015. Se utilizó una prevalencia de EPI de $40 \%$, un margen de error del $5 \%$ y un nivel de confianza del 95\%. Las madres de los niños fueron seleccionadas por muestreo probabilístico aleatorio simple durante actividades de promoción de salud bucal y atención odontológica realizadas en una Jornada de Salud.

\section{Criterios de inclusión y exclusión}

Se consideraron como criterios de inclusión: ser madre de niños entre 0 meses a 5 años, ser nativas de la comunidad y aceptar su participación en el estudio mediante consentimiento informado; fueron excluidas madres que poseían discapacidad cognitiva o alguna alteración que pudiera interferir en la respuesta.

\section{Instrumento}

Se aplicó cuestionario autosuministrado, previamente probado en población con características similares a la población de estudio (50 cuestionarios), utilizando el instrumento de investigaciones operativas en AIEPI que incluía: 1. Variables sociodemográficas: sexo (masculinofemenino) y edad del infante ( $<2$ meses; $\geq 2$ meses5 años), edad de la madre ( $\leq 19$ años, 20-44 años), nivel de escolaridad madre/cuidador ( $\geq 10$ años de estudio, $<10$ años de estudio), número de hijos ( $\leq 2$ hijos; >2 hijos), convivencia con esposo o abuela del menor, tipo de servicio de salud (público-privado), 2. Presencia de enfermedades prevalentes en la infancia (EPI) según estrategia AIEPI en niños menores de 5 años (si-no): enfermedad febril, resfriado, bronquitis, neumonía, insuficiencia respiratoria aguda (IRA), diarrea, desnutrición, dengue, enfermedad inmunoprevenible, caries dental, otitis y otras enfermedades ${ }^{4}$. 3. Prácticas Claves según estrategia AIEPI realizadas en el niño: lactancia materna ( $\leq 12$ meses, 13-23 meses, $\geq 24$ meses) ${ }^{20}$, uso de biberón, dormir con biberón, cepillado bucal después de mamar o tomar biberón y antes de dormir, frecuencia de cepillado, lavado de manos, poseer carnet de crecimiento y desarrollo y esquema de vacunación completo para la edad.

\section{Análisis de los datos}

En el análisis de los datos se utilizó el programa Microsoft Office Excel 2016 ${ }^{\circledR} \quad$ (Microsoft Corporation, Redmond, Washington, USA) para realizar pruebas de estadística descriptiva y determinar prevalencia de enfermedades $y$ 
frecuencias de las prácticas preventivas. Se realizó Regresión de Poisson en STATA versión 11.2 (Stata Corp, College Station, TX, USA) para establecer asociación entre EPI y las variables sociodemográficas y prácticas claves AIEPI con intervalo de confianza de $95 \%$ y significancia estadística.

\section{Declaración de los aspectos éticos}

El estudio se realizó según disposiciones éticas estipuladas en resolución 8430 de 1993 del Ministerio de Salud de la República de Colombia y Declaración de Helsinki (Modificación de Edimburgo, 2000) y aprobación del Comité de ética del Departamento de Investigaciones de la Universidad de Cartagena.

\section{RESULTADOS}

Las características sociodemográficas de la población se encuentran en la Tabla 1.

\section{Prevalencia de enfermedades en la Infancia}

Con respecto a la prevalencia de EPI en niños entre 0 meses y 5 años, $84,3 \%$ de los niños presentaba alguna enfermedad. Las enfermedades más prevalentes en los infantes fueron resfriado $(62,0 \%$ IC 95\%: 57,9-66,1), caries dental $(24,8 \%$ IC $95 \%$ : $21,1-28,4)$ y diarrea $(19,5 \%$ IC $95 \%: 16,1-22,8)$. No obstante, 65 menores presentaban bronquitis (11,9\% IC 95\%: 9,1-14,5) y 43 presentaron IRA (7,9\% IC 95\%: 5,5-10,1) (Tabla 2).

Tabla 1. Características sociodemográficas de binomios madres-niños menores de 5 años, habitantes de Boquilla, Tierra Baja, Manzanillo y Caño del Oro, de Cartagena, 2014 -2015.

\begin{tabular}{|c|c|c|}
\hline Variables & Subescalas & $\mathbf{n}(\%)$ \\
\hline \multirow{2}{*}{ Edad del niño } & $<2$ meses & $13(2,4)$ \\
\cline { 2 - 3 } & $\geq 2$ meses y hasta 5 años & $535(97,6)$ \\
\hline \multirow{2}{*}{ Sexo del niño } & Femenino & $278(50,7)$ \\
\cline { 2 - 3 } & Masculino & $270(49,3)$ \\
\hline \multirow{2}{*}{ Edad madre/cuidador } & $\leq 19$ años & $56(10,2)$ \\
\cline { 2 - 3 } Nivel de escolaridad madre/cuidador & $20-44$ años & $492(89,8)$ \\
\cline { 2 - 3 } & $<10$ años de estudio & $303(55,3)$ \\
\hline \multirow{2}{*}{ Tipos de servicios de salud } & $<10$ años de estudio & $245(44,7)$ \\
\cline { 2 - 3 } & Público & $476(86,8)$ \\
\hline \multirow{2}{*}{ Número de hijos } & Privado & $72(13,2)$ \\
\hline \multirow{2}{*}{ Convivencia con el esposo } & $1-2$ hijos & $392(71,5)$ \\
\cline { 2 - 3 } & $\geq 3$ hijos & $156(28,5)$ \\
\hline \multirow{2}{*}{ Convivencia con la abuela del hijo } & Sí & $428(78,1)$ \\
\cline { 2 - 3 } & No & $120(21,9)$ \\
\hline
\end{tabular}


Tabla 2. Prevalencia de enfermedades en niños menores de 5 años habitantes de Boquilla, Tierra Baja, Manzanillo y Caño del Oro, de Cartagena, 2014 -2015.

\begin{tabular}{|c|c|c|}
\hline \multirow{2}{*}{ Variable } & Presenta & No presenta \\
\hline & n ( \%) & n ( \%) \\
\hline Presenta alguna enfermedad? & $479(87,5)$ & $69(12,5)$ \\
\hline Enfermedad Febril & $18(3,3)$ & $530(96,7)$ \\
\hline IRA & $43(7,9)$ & $505(92,1)$ \\
\hline Resfriado & $339(62,0)$ & $209(38,0)$ \\
\hline Bronquitis & $65(11,9)$ & $483(88,1)$ \\
\hline Neumonía & $6(1,1)$ & $542(98,9)$ \\
\hline Diarrea & $107(19,5)$ & $441(80,5)$ \\
\hline Dengue & $4(0,7)$ & $544(99,3)$ \\
\hline Inmunoprevenibles & $3(0,7)$ & $545(94,3)$ \\
\hline Otitis & $8(1,5)$ & $540(98,5)$ \\
\hline Desnutrición & $10(1,8)$ & $538(98,2)$ \\
\hline Caries dental & $136(24,8)$ & $412(75,2)$ \\
\hline \multicolumn{3}{|l|}{ Otras Enfermedades } \\
\hline Enfermedad en piel & $5(1,0)$ & $543(99,0)$ \\
\hline Parasitosis & $1(0,2)$ & $547(99,8)$ \\
\hline Infección urinaria & $1(0,2)$ & $547(99,8)$ \\
\hline Enfermedad hepática & $2(0,4)$ & $546(99,6)$ \\
\hline
\end{tabular}

\section{Prácticas clave}

El $80,5 \%$ y el $74,5 \%$ practicó la lactancia materna durantelos primeros seis meses y primer año de vida del infante, respectivamente. La duración total de lactancia materna practicada fue de 10 meses; solo el $39,3 \%$ de los niños tomaban biberón y $15,3 \%$ dormían con el biberón en la boca. En relación con hábitos de salud bucal, al $83,2 \%$ de los niños no se les realizaba cepillado bucal después de tomar biberón o mamar y al 55,3\% no le cepillaban los dientes antes de dormir. Otras prácticas claves se hallan en la Tabla 3.
EPI, Variables Sociodemográficas y Prácticas clave

Al asociar presencia de EPI con variables sociodemográficas y prácticas clave, se encontró asociación con significancia estadística con el tipo de servicio de salud $(p=0,032)$ (Tabla 4$)$, no cepillado dental después de tomar el biberón o mamar, y antes de dormir con valores de $p \leq 0,05$. (Tabla 5). 
Tabla 3. Prácticas Preventivas de las Madres relacionadas con Prevención de Enfermedades Prevalentes de la Infancia en niños menores de 5 años habitantes de Boquilla, Tierra Baja, Manzanillo y Caño del Oro, de Cartagena, $2014-2015$.

\begin{tabular}{|c|c|c|}
\hline Variables & Subescalas & n (\%) \\
\hline \multirow{3}{*}{ Lactancia materna } & $<12$ meses & $408(74,5)$ \\
\hline & 13-23 meses & $107(19,5)$ \\
\hline & $\geq 24$ meses & $33(6,0)$ \\
\hline \multirow{2}{*}{ Lactancia exclusiva } & 6 meses & $441(80,5)$ \\
\hline & $<6$ meses & $107(19,5)$ \\
\hline \multirow{2}{*}{ El niño toma tetero } & Sí & $215(39,3)$ \\
\hline & No & $333(60,7)$ \\
\hline \multirow{2}{*}{$\begin{array}{l}\text { El niño duerme con el tetero en la } \\
\text { boca }\end{array}$} & Sí & $84(15,3)$ \\
\hline & No & $464(84,7)$ \\
\hline \multirow{2}{*}{$\begin{array}{c}\text { El niño es cepillado después de } \\
\text { tomar el tetero o mamar }\end{array}$} & Sí & $89(16,8)$ \\
\hline & No & $459(83,2)$ \\
\hline \multirow{2}{*}{ Cepillado dental antes de dormir } & Sí & $245(44,7)$ \\
\hline & No & $303(55,3)$ \\
\hline \multirow{2}{*}{$\begin{array}{l}\text { Cuantas veces al día le cepillan los } \\
\text { dientes al niño }\end{array}$} & $1 \mathrm{vez}$ & $154(28,2)$ \\
\hline & $2-3$ veces & $394(71,8)$ \\
\hline \multirow{2}{*}{ Lavados de manos al niño } & Sí & $478(87,2)$ \\
\hline & No & $70(12,8)$ \\
\hline \multirow{2}{*}{ Carnet de Crecimiento y Desarrollo } & Sí & $496(90,8)$ \\
\hline & No & $52(9,2)$ \\
\hline \multirow{2}{*}{ Esquema de vacunación completo } & Sí & $512(93,4)$ \\
\hline & No & $36(6,6)$ \\
\hline
\end{tabular}


Tabla 4. Asociación entre variables sociodemográficas de las madres con presencia de enfermedades en niños menores de 5 años, habitantes de Boquilla, Tierra Baja, Manzanillo y Caño del Oro, de Cartagena, 2014 -2015.

\begin{tabular}{|c|c|c|c|c|}
\hline \multirow{2}{*}{ Variables } & \multicolumn{4}{|c|}{ Enfermedad Prevalente de la Infancia } \\
\hline & Ausencia & Presencia & RP (IC 95\%) & $\mathbf{p}$ \\
\hline \multicolumn{5}{|l|}{ Edad del niño } \\
\hline$<2$ meses & $5(0,91)$ & $8(1,4)$ & $1,43(0,92-2,20)$ & 0,104 \\
\hline$\geq 2$ meses y hasta 5 años & $64(11,6)$ & $471(85,9)$ & & \\
\hline \multicolumn{5}{|l|}{ Sexo del niño } \\
\hline Femenino & $32(5,8)$ & $246(45,0)$ & $1,02(0,96-1,09)$ & 0,440 \\
\hline Masculino & $37(6,7)$ & $233(42,5)$ & & \\
\hline \multicolumn{5}{|l|}{ Edad madre } \\
\hline$\leq 19$ años & $5(0,91)$ & $50(9,12)$ & $1,04(0,95-1,14)$ & 0,343 \\
\hline 20-44 años & $64(11,7)$ & $429(78,0)$ & & \\
\hline \multicolumn{5}{|c|}{ Nivel escolaridad madre/cuidador } \\
\hline$\geq 10$ años de estudio & $39(7,1)$ & $263(48,0)$ & & \\
\hline$<10$ años de estudio & $30(5,5)$ & $216(39,4)$ & $0,99(0,93-1,05)$ & 0,800 \\
\hline \multicolumn{5}{|l|}{ Número de hijos } \\
\hline 1-2 hijos & $51(9,3)$ & $340(62,0)$ & & \\
\hline$\geq 3$ hijos & $18(3,3)$ & $139(25,3)$ & $0,98(0,91-1,05)$ & 0,605 \\
\hline \multicolumn{5}{|l|}{ Convivencia con esposo } \\
\hline Sí & $55(10,0)$ & $373(65,7)$ & & \\
\hline No & $14(2,55)$ & $106(19,3)$ & $0,98(0,91-1,06)$ & 0,723 \\
\hline \multicolumn{5}{|l|}{ Convivencia con abuela } \\
\hline Sí & $36(6,5)$ & $207(37,7)$ & & \\
\hline No & $33(8,0)$ & $272(49,6)$ & $1,04(0,98-1,11)$ & 0,170 \\
\hline \multicolumn{5}{|l|}{ Tipo de Servicio de Salud } \\
\hline Público & $52(9,5)$ & $423(77,2)$ & $1,16(1,02-1,33)$ & $0,023^{*}$ \\
\hline Privado & $17(3,1)$ & $55(10,2)$ & & \\
\hline
\end{tabular}

*Valores de $\mathrm{p}$ significantes 
Tabla 5. Asociación entre prácticas clave de AIEPI aplicadas por las madres con presencia de enfermedades en niños menores de 5 años, habitantes de Boquilla, Tierra Baja, Manzanillo y Caño del Oro, 2014 -2015.

\begin{tabular}{|c|c|c|c|c|}
\hline \multirow[t]{2}{*}{ Variables } & \multicolumn{4}{|c|}{ Enfermedad Prevalente de la Infancia } \\
\hline & Ausencia & Presencia & RP (IC $95 \%)$ & $\mathbf{p}$ \\
\hline \multicolumn{5}{|l|}{ Lactancia materna } \\
\hline Sí & $63(11,5)$ & $440(80,3)$ & & \\
\hline No & $6(1,1)$ & $39(7,1)$ & $1,00(0,89-1,13)$ & 0,879 \\
\hline \multicolumn{5}{|l|}{ El niño toma tetero } \\
\hline Sí & $24(4,4)$ & $191(34,8)$ & $1,02(0,96-1,09)$ & 0,409 \\
\hline No & $45(8,2)$ & $288(52,5)$ & & \\
\hline \multicolumn{5}{|c|}{ El niño duerme con el tetero en la boca } \\
\hline Sí & $12(2,2)$ & $72(13,1)$ & $0,97(0,88-1,07)$ & 0,630 \\
\hline No & $57(10,4)$ & $407(74,2)$ & & \\
\hline \multicolumn{5}{|c|}{$\begin{array}{l}\text { El niño es cepillado después de tomar el } \\
\text { tetero o mamar }\end{array}$} \\
\hline Sí & $6(1,1)$ & $83(15,1)$ & & \\
\hline No & $63(11,5)$ & $396(72,2)$ & $1,08(1,01-1,15)$ & $0,022^{*}$ \\
\hline \multicolumn{5}{|c|}{ Cepillado dental antes de dormir } \\
\hline Sí & $42(7,7)$ & $203(37,0)$ & & \\
\hline No & $27(4,9)$ & $276(50,3)$ & $0,90(0,85-0,97)$ & $0,006^{*}$ \\
\hline \multicolumn{5}{|c|}{$\begin{array}{l}\text { Cuantas veces al día se le cepillan los } \\
\text { dientes al niño }\end{array}$} \\
\hline $1 \mathrm{vez}$ & $16(2,9)$ & $138(25,2)$ & $1,03(0,96-1,10)$ & 0,305 \\
\hline $2-3$ veces & $53(9,7)$ & $341(62,2)$ & & \\
\hline \multicolumn{5}{|l|}{ Lavado de manos al niño } \\
\hline Sí & $62(11,3)$ & $416(75,9)$ & & \\
\hline No & $7(1,3)$ & $63(11,5)$ & $0,96(0,88-1,05)$ & 0,442 \\
\hline \multicolumn{5}{|c|}{ Carnet de crecimiento y desarrollo } \\
\hline Sí & $64(11,7)$ & $434(79,1)$ & & \\
\hline No & $5(0,9)$ & $45(8,2)$ & $0,96(0,87-1,06)$ & 0,522 \\
\hline \multicolumn{5}{|c|}{$\begin{array}{l}\text { Esquema de vacunación completo para la } \\
\text { edad }\end{array}$} \\
\hline Sí & $65(11,9)$ & $447(81,5)$ & & \\
\hline No & $4(0,7)$ & $32(5,8)$ & $0,98(0,87-1,10)$ & 0,769 \\
\hline
\end{tabular}

*Valores de $\mathrm{p}$ significantes

\section{DISCUSIÓN}

A través del presente estudio se logra evidenciar cómo desde la consulta odontológica se realiza un abordaje integral en la atención de la primera infancia, identificando, además de problemas de salud bucal, prevalencias de otras enfermedades y su asociación con prácticas claves promovidas por la Estrategia AIEPI en su componente comunitario, como las prácticas de higiene bucal. Esto obligaría al profesional en odontología a conocer signos y síntomas de riesgo de las demás enfermedades prevalentes en este rango de edad para remitir e intervenir oportunamente, constituyéndose como un recurso humano valioso para la ejecución de la estrategia AIEPI.

Con respecto a las prevalencias de las enfermedades reportadas en el presente estudio, el resfriado (62\%) corresponde a la enfermedad más prevalente Esto es similar a lo reportado en niños menores de 5 años de una comunidad afro-descendiente del Caribe 
Colombiano ${ }^{17}$, único estudio que permite su comparación al coincidir en las poblaciones estudiadas respecto a la ubicación geográfica a orillas del mar Caribe, lo que propicia la aparición frecuente de episodios gripales y sus complicaciones como la IRA. En el 2018, en la ciudad de Cartagena fue reportada una tasa de mortalidad en niños menores de 5 años por enfermedad respiratoria aguda de 13,9 fallecidos por cada 100.000 infantes, aumentando el número de muertes por IRA de 11 casos a 34 casos en un año del 2017 al $2018^{19}$. Como se aprecia, estas enfermedades constituyen una de las primeras causas de muertes en esta población y un desafío para el planteamiento de políticas públicas y programas preventivos que permitan controlar la aparición de nuevos casos en la ciudad, desde la participación de todos los profesionales de la salud, incluidos los odontólogos quienes atienden a este grupo etario.

La segunda enfermedad más prevalente reportada en el estudio corresponde a la caries dental (24,8\%) por encima de la diarrea. Aunque esta cifra resulta inferior a la reportada en Colombia para primera infancia $(33,84 \%)^{21}$, se confirma en este estudio cómo la caries dental sigue siendo una de las enfermedades prevalentes de la infancia, como lo ilustra la Estrategia AIEPI, y es considerada un problema de salud pública al comprometer la calidad de vida, el crecimiento y desarrollo del niño, y la dinámica familiar de forma general ${ }^{20,22}$. Esto evidencia la importancia de la atención integral en estas poblaciones al valorar también su salud bucal como lo propone la estrategia ${ }^{1}$.

La prevalencia de caries dental en los infantes ha sido asociada con factores como la baja escolaridad de los cuidadores, el inicio tardío de hábitos de higiene bucal, el alto consumo de carbohidratos y la baja demanda de programas preventivos ${ }^{23,24}$. Probablemente, la presencia de caries dental en este estudio estuvo relacionada con la duración de la lactancia materna, teniendo en cuenta que el $25 \%$ de los niños del estudio la gozaron por más de 12 meses, pero al realizar asociación de estas variables no se hallaron datos con significancia estadística. Algunos estudios indican que un tiempo de lactancia superior a los 12 meses y la lactancia nocturna se encuentran asociadas a 2-3 veces el incremento de caries dental en dentición decidua, posiblemente debido a inadecuada higiene bucal y al consumo de alimentos con altos contenidos de azúcares ${ }^{25,26}$.

En este sentido, es necesario que los odontólogos anticipadamente dediquen esfuerzos para promover prácticas adecuadas de salud bucal por parte de los padres y/o cuidadores, educando a la madre y su familia desde el embarazo y acompañándola durante todo su proceso de lactancia hasta el destete, enseñando de manera temprana las recomendaciones en relación con el inicio de cepillado bucal en primera infancia, uso de cremas dentales con flúor de 1000-1500 ppm, tal y como es recomendado por la $\mathrm{OMS}^{27}$. Así mismo, considerando que los alimentos consumidos por la madre son transferidos a través del líquido amniótico y la leche materna, se pueden ofrecer orientaciones con relación a una dieta baja en azúcares que apoyen la modificación de las preferencias alimenticias del niño hacia el inicio de la alimentación complementaria, que desestimulen su consumo y ayuden a prevenir al mismo tiempo caries dental, diabetes y obesidad, generando un gran impacto para la salud pública ${ }^{28,29}$.

Además, se observó que en promedio el 74,4 \% de las madres practicó la lactancia materna durante los primeros 12 meses de vida y la media de duración total de esta práctica fue de 10 meses. Sin embargo, en Colombia solo el $52,2 \%{ }^{30}$ de los niños continuó la lactancia materna hasta el primer año de vida y la media de duración total fue de 14,9 meses $^{31}$. Por lo tanto, se evidencia una práctica adecuada en medio de la vulnerabilidad de la población y se confirma que esta práctica es más realizada en países de bajos y medianos ingresos que en países ricos ${ }^{31}$. En los infantes, la lactancia materna actúa como un factor protector frente a la aparición y muerte por enfermedades infecciosas, ya que aporta vitaminas, minerales, propiedades inmunológicas y antiinflamatorias, constituyéndose casi que en un alimento personalizado para sus necesidades ${ }^{32}$. Adicionalmente, aporta beneficios a las madres al disminuir la incidencia de cáncer de mama y de ovario, siendo el único alimento que protege de forma bidireccional y simultanea al binomio madrehijo ${ }^{33}$. Por tanto, se propone que en los países de bajos recursos se aumenten los esfuerzos en el diseño de estrategias que fomenten la lactancia 
materna, considerando que estos países presentan altas prevalencias de cáncer de mama ${ }^{31}$.

Con respecto a las bajas prevalencias reportadas para la diarrea, al ser comparadas con las evidenciadas para estas poblaciones en otros estudios $^{2}$, es importante destacar que dichas cifras demuestran la efectividad de prácticas preventivas como el lavado de manos y la incorporación de la vacuna contra el Rotavirus. La comunidad estudiada presenta factores de riesgo locales para la presencia de diarrea como altos índices de pobreza y hacinamiento, deficiencias en el saneamiento básico, ausencia de alcantarillado y acueducto, consumo de aguas no tratadas, además de contar con cuidadores con bajo nivel de escolaridad, factores que han sido asociados a las prevalencias de estas enfermedades ${ }^{34-36}$. Los casos de diarrea presentados se podrían deber a la presencia de los factores de riesgo reportados para su aparición. El conocimiento por parte del odontólogo sobre estos factores que pueden comprometer la salud de los pacientes ayudaría en su control, especialmente durante los trabajos comunitarios a través de estrategias como la consejería. Consecuentemente, se encontró que la mayoría de los niños presentaban Carnet de Crecimiento y Desarrollo y vacunas completas para la edad. En la literatura, estas prácticas se han asociado con la disminución de la mortalidad infantil e incidencia de enfermedades inmunoprevenibles ${ }^{37,38}$, y fue identificada como factor protector para el desarrollo del menor ${ }^{38}$; por tanto, se resalta la importancia de solicitarlo en todas las consultas de salud, independientemente del motivo.

Al asociar las enfermedades prevalentes en la infancia con las variables sociodemográficas se halló que el contar con servicios públicos de salud representa un riesgo para su presencia. Las poblaciones estudiadas en su mayoría poseen servicios de salud público y el centro de salud más cercano a los corregimientos de la Boquilla, Tierra Baja y Caño del Oro se encuentra a una distancia de $1 \mathrm{~km}$ de la ciudad de Cartagena. Por lo tanto, los habitantes presentan algunas veces dificultades en el acceso a servicios médicos y odontológicos, lo que limita su participación en programas de prevención y educación en salud y los hace más propensos al desarrollo de enfermedades, evidenciándose una situación de vulnerabilidad e inequidad en salud. En este sentido, un estudio realizado en varias ciudades de Colombia mostró que, a pesar de las notables mejorías en los servicios de la salud, se continúan presentando no solo barreras geográficas sino también de ofertas culturales y administrativas, lo que continúa causando dificultades en el acceso a los servicios de salud y aumenta la vulnerabilidad de los grupos poblacionales ${ }^{39}$.

También se halló asociación entre la presencia de enfermedad en los niños e inadecuadas prácticas de madres y/o cuidadoras, como no cepillar al infante después de tomar el biberón o mamar, o antes dormir. De esta forma, inadecuadas prácticas de salud bucal en infantes estarían asociadas con mayor presencia de enfermedad, posiblemente relacionada con insuficiente educación en salud bucal, deficiencias en la autoeficacia de los cuidadores y determinantes de la salud general en relación con hábitos de cuidado. No obstante, la respuesta por parte de las madres y/cuidadores de la frecuencia de cepillado podría estar influenciada por comportamientos socialmente deseados y puede que no necesariamente coincida con la frecuencia real de cepillado de los niños ${ }^{40}$. Consecuentemente, los resultados en salud de los niños pueden estar influenciados por factores psicosociales, creencias, conocimientos y actitudes de los cuidadores que interfieren en la aplicación de comportamientos adecuados en salud ${ }^{40}$.

Al respecto, un estudio reporta que todos los factores anteriormente mencionados y la autoeficacia de los cuidadores fue un factor predictor para la implementación de buenos hábitos de salud bucal en los niños ${ }^{40}$, situación contraria a la presentada en este estudio. Por tanto, se puede observar la multiplicidad de factores involucrados en la aplicación de correctos hábitos de salud bucal y cómo estos podrían estar relacionados con la implementación de cuidados generales en salud que actuarían como factores protectores para la adquisición de enfermedades en los menores ${ }^{41,42}$. Los odontólogos, al identificar niños con enfermedades bucales y hábitos de higiene bucal, desde su consulta o en actividades comunitarias, deben indagar por la presencia de otras EPI. 
Probablemente los cuidadores enfoquen su atención en el tratamiento de las enfermedades que esté presentando el niño y en el suministro de medicamentos y alimentos tal y como lo propone una de las prácticas claves de AIEPI, y por eso descuiden los hábitos de higiene bucal del infante. Por eso, debe hacerse énfasis en esta práctica durante la enfermedad del niño, incluso desde la consulta médica. Además, el odontólogo también debe enfatizar la importancia de la higiene bucal en los momentos de enfermedad del niño, evitando futuras sobreinfecciones y complicaciones. En este sentido, se evidencia la necesidad del trabajo en equipo y multidisciplinario para la adecuada atención de la primera infancia.

En términos generales, la presente investigación sugiere un cambio en la atención prestada por el odontólogo desde la integralidad, articulando la atención en salud bucal a la detección de enfermedades prevalentes en la infancia y el fomento en la promoción de las prácticas claves, como lo recomienda la Estrategia AIEPI, con el fin de contribuir a la disminución de la morbimortalidad en estas poblaciones. Así, este logra ser, a la luz de los autores, el primer estudio que articula la participación y el rol de los odontólogos en la implementación de la estrategia. Finalmente, madres y/o cuidadores, líderes comunitarios, profesores, agentes de salud y todo el personal alrededor de las familias durante la primera infancia deben ser capacitados por equipos multidisciplinarios que promuevan la aplicación de las prácticas protectoras de la estrategia AIEPI y permitan el mejoramiento de los hábitos de cuidado de salud general y bucal en la primera infancia a través del empoderamiento de la población.

\section{CONCLUSIÓN}

La prevalencia de enfermedades en la infancia como resfriado, caries dental y diarrea fue alta en niños menores de 5 años; pertenecer al servicio de salud público y no cepillar los dientes de los niños después de tomar el biberón, mamar o antes de dormir se comportaron como factores de riesgo para la presencia de estas enfermedades. Es necesario articular la presencia del odontólogo en la ejecución de estrategias que promueven trabajos de atención integral a la primera infancia, como lo propone la Estrategia AIEPI, a través de equipos multidisciplinarios que aporten a la disminución de las inequidades en salud en comunidades vulnerables.

\section{AGRADECIMIENTOS}

A las comunidades de Boquilla, Tierra Baja y Caño del Oro por su colaboración en la recolección de la información y a los estudiantes de odontología que participaron en las actividades asistenciales.

\section{DECLARACIÓN SOBRE CONFLICTO DE INTERESES}

Los autores no reportan conflictos a declarar en el presente estudio.

\section{CONTRIBUCIÓN DE LOS AUTORES}

Primer autor: diseño metodológico, trabajo de campo, análisis estadístico, redacción y revisión crítica del artículo.

Segundo autor: análisis estadístico, redacción y revisión crítica del artículo.

Tercer autor: diseño metodológico, redacción y revisión crítica del artículo.

\section{REFERENCIAS BIBLIOGRÁFICAS}

1. González R. Salud materno-infantil en las Américas. Rev. chil. obstet. Ginecol. 2010; 75(6):411-21. Doi: https://dx.doi.org/10.4067/S071775262010000600011

2. Ministerio de Salud y Protección social de Colombia-Organización Panamericana de la Salud. Libro Clínico Atención Integral a Enfermedades de la Primera Infancia. Colombia: OPS; 2012. [internet]. [Consultado 2017 Ago 20]. Disponible en: http://www.minsalud.gov.co/sites/rid/Lists/Bibliot ecaDigital/RIDE/VS/PP/guia-facilitador-atencionintegrada-enfermedades.pdf.

3. Organización Mundial de la Salud. ODM: reducir la mortalidad infantil. Ginebra 2016. [internet]. [Consultado 2017 Ago 20]. Disponible en: 
http://www.who.int/topics/millennium_developm ent_goals/child_mortality/es/.

4. Organización Mundial de la Salud. Estadísticas Sanitarias Mundiales. Ginebra 2010. [internet]. [Consultado 2017 Ago 20]. Disponible en: http://www.who.int/whosis/whostat/ES_WHS10_F ull.pdf.

5. Mashal T, Takano T, Nakamura K, Kizuki M, Hemat $S$, Watanabe $\mathrm{M}$ et al. Factors associated with the health and nutritional status of children under 5 years of age in Afghanistan: family behaviour related to women and past experience of war-related hardships. BMC Public Health. 2008; 8: 301. Doi: http://doi.org/10.1186/1471-2458-8-301.

6. Bauman LJ, Silver EJ, Stein REK. Cumulative social disadvantage and children's health. Pediatrics. 2006. 117:1321-3128. Doi: http://doi.org/ 10.1542/peds.2005-1647

7. Vishwanathaiah, S. Knowledge, Attitudes, and Oral Health Practices of School Children in Davangere. Int J Clin Pediatr Dent. 2016; 9(2): 17276. Doi: http://doi.org/10.5005/jp-journals-100051358

8. Pan American Health Organization, World Health Organization. Integrated Management of Childhood Illness (IMCl). Final report of the 26th Pan American Sanitary Conference. [internet]. [Consultado 2017 Ago 20]. Disponible en: https://www.paho.org/english/gov/csp/csp26.r10e.pdf?ua=1

9. Gonzales M, Quiroga C, Pantoja M, Cornejo C, Mendoza A. Experiencia con la estrategia AIPEI en el pregrado de medicina de la Universidad Mayor de San Andrés. Rev. Bol. Ped. 2002; 41(1): 3-6. [internet]. [Consultado 2017 Ago 20]. Disponible en: http://www.scielo.org.bo/scielo.php?script=sci_art text\&pid=S1024-

06752002000100002\&lng=es \&nrm=iso>.

10. Cárdenas LM, Cotes $K$, Chaparro PE, Fernández JA, Paternina A, Castañeda C, et al. Maternal mortality in Colombia in 2011: a two level ecological study. PLoS One. 2015; 10(3):1-10. Doi: 10.1371/journal.pone.0118944.

11. Nguyen DT, Leung KK, McIntyre L, Ghali WA, Sauve R. Does integrated management of childhood illness $(\mathrm{IMCl})$ training improve the skills of health workers? A systematic review and meta-analysis. PLoS One. 2013; 12;8(6):e66030. Doi: 10.1371/journal.pone. 0066030 .

12. Jonker L, Stellenberg EL. Missed opportunities in child healthcare. Afr J Prim Health Care Fam Med. 2014;5;6(1):E1-7. Doi: 10.4102/phcfm.v6i1.537.

13. Amaral J, Gouws E, Bryce J, Leite AJ, Cunha AL, Victora CG. Effect of Integrated Management of Childhood Illness (IMCI) on health worker performance in Northeast-Brazil. Cad Saude Publica. 2004; 20 Suppl 2:S209-19. Doi: 10.1590/s0102$311 \times 2004000800016$

14. Uzochukwu B, Onwujekwe O, Ezeilo E, Nwobi E, $\mathrm{Ndu} A$, et al. Integrated management of childhood illness in Nigeria: Does short-term training of health workers improve their performance? Public Health 2008; 122: 367-370. Doi: 10.1016/j.puhe.2007.07.001

15. Anand K, Patro BK, Paul E, Kapoor SK. Management of sick children by health workers in Ballabgarh: lessons for implementation of $\mathrm{IMCl}$ in India. J Trop Pediatr. 2004;50(1):41-7. Doi: 10.1093/tropej/50.1.41

16. Huicho L, Scherpbier RW, Nkowane AM, Victora CG; Multi-Country Evaluation of IMCI Study Group. How much does quality of child care vary between health workers with differing durations of training? An observational multicountry study Lancet. 2008;13;372(9642):910-6. Doi: 10.1016/S01406736(08)61401-4.

17. Díaz S, Arrieta K, Ramos K. AlEPI y prácticas maternas frente a la diarrea y resfriado común en afrodescendientes del caribe colombiano. Rev. salud pública. 2013; 15 (4): 555-564. [internet]. [Consultado 2017 Sep 27]. Disponible en: http: //revistas.unal.edu.co/index.php/revsaludpublica/a rticle/view/33842

18. Zaman S, Ashraf RN, Martines J. Training in complementary feeding counselling of healthcare workers and its influence on maternal behaviours and child growth: a cluster-randomized controlled trial in Lahore, Pakistan. J Health Popul Nutr. 2008; 26(2):210-22. [internet]. [Consultado 2017 Sep 28]. Disponible en: https://www.ncbi.nlm.nih.gov/pmc/articles/PMC27 40673/ 
19. Departamento Administrativo Distrital de Salud. Salud: Informe de Calidad Cartagena como vamos 2018. [internet].Disponible en: http://www.cartagenacomovamos.org/nuevo/wpcontent/uploads/2019/09/Informe_de_Calidad_de _Vida-2018_Cartagena_C\%C3\%B3mo_Vamos.pdf

20. Naidu R, Nunn J, Donnelly-Swift E. Oral healthrelated quality of life and early childhood caries among preschool children in Trinidad. BMC Oral Health. 2016; 16: $128 . \quad$ Doi: http://doi.org/10.1186/s12903-016-0324-7

21. Ministro de Salud y Protección Social de Colombia. IV ESTUDIO NACIONAL DE SALUD BUCAL (ENSAB IV) Situación en Salud bucal. Colombia; 2014. [internet]. [Consultado 2020 Ago 02]. Disponible en: https://www.minsalud.gov.co/sites/rid/Lists/Bibliot ecaDigital/RIDE/VS/PP/ENSAB-IV-Situacion-BucalActual.pdf

22. Martignon S, Pitts NB, Goffin G, et al. CariesCare practice guide: consensus on evidence into practice [published correction appears in $\mathrm{Br}$ Dent J. 2019 Dec;227(11):988]. Br Dent J. 2019;227(5):353-362. Doi:10.1038/s41415-019-0678-8

23. Anil S, Anand PS. Early Childhood Caries: Prevalence, Risk Factors, and Prevention. Frontiers in Pediatrics. 2017; 18(5):157. Doi: http://doi.org/10.3389/fped.2017.00157

24. Nota A, Darvizeh A, Primožič J, et al. Prevalence of Caries and Associated Risk Factors in a Representative Group of Preschool Children from an Urban Area with High Income in Milan Province, Italy. Int J Environ Res Public Health. 2020;17(10):3372. Doi:10.3390/ijerph17103372

24. Peres KG, Nascimento GG, Peres MA, Mittinty MN, Demarco FF, Santos IS et al. Impact of Prolonged Breastfeeding on Dental Caries: A Population-Based Birth Cohort Study. Pediatrics. 2017; 140(1): e20162943. Doi: http://doi.org/10.1542/peds.2016-2943

25. Victora CG, Bahl R, Barros AJ, França GV, Horton S, Krasevec J, Murch S,Sankar MJ, Walker N, Rollins NC; Lancet Breastfeeding Series Group. Breastfeeding in the 21st century: epidemiology, mechanisms, and lifelong effect. Lancet. 2016; 387(10017):475-90.

Doi: http://doi.org/10.1016/S0140-6736(15)01024-7

26. World Health Organization. Ending childhood dental caries: WHO implementation manual. Geneva; 2019. [internet]. [Consultado 2020 Ago 02]. Disponible en: https://apps.who.int/iris/handle/10665/330643.

27. Mennella JA, Jagnow CP, Beauchamp GK. Prenatal and postnatal flavor learning by human infants. Pediatrics. 2001;107(6):E88-E93. Doi:10.1542/peds.107.6.e88

28. Schaal B, Marlier L, Soussignan R. Human foetuses learn odours from their pregnant mother's diet. Chem Senses. 2000;25(6):729-737. Doi:10.1093/chemse/25.6.72

29. Ministro de Salud y Protección Social de Colombia. Encuesta nacional de la situacion nutricional ENSIN. 2015. Colombia; 2015. [internet]. [Consultado 2020 Ago 02]. Disponible en: https://www.minsalud.gov.co/sites/rid/Lists/Bibliot ecaDigital/RIDE/VS/ED/GCFI/ensin-colombia2018.pdf

30. Roberts TJ, Carnahan E, Gakidou E. Can breastfeeding promote child health equity? A comprehensive analysis of breastfeeding patterns across the developing world and what we can learn from them. BMC Med. 2013; 11: 254. Doi: http://doi.org/10.1186/1741-7015-11-254

31. Hooper LV, Littman DR, Macpherson AJ. Interactions between the microbiota and the immune system. Science. 2012; 336: 1268-73. Doi: http://doi.org/10.1126/science.1223490

32. Mayer EA, Knight R, Mazmanian SK, Cryan JF, Tillisch K. Gut microbes and the brain: paradigm shift in neuroscience. J Neurosci. 2014; 34: 15490-15496. Doi: $\quad$ http://doi.org/10.1523/JNEUROSCl.329914.2014

33. Kosek M, Yori PP, Pan WK, Olortegui MP, Gilman $\mathrm{RH}$, Perez J, et al. Epidemiology of highly endemic multiply antibiotic-resistant shigellosis in children in the Peruvian Amazon. Pediatrics. 2008; 122:e541e9. Doi: http://doi.org/10.1542/peds.2008-0458 
34. Tornheim JA, Morland KB, Landrigan PJ, Cifuentes $\mathrm{E}$. Water privatization, water source, and pediatric diarrhea in Bolivia: epidemiologic analysis of a social experiment. Int J Occup Environ Health. 2009; 15: 241-248. Doi: http://dx.doi.org/10.1179/oeh.2009.15.3.241

35. Aziz FAA, Ahmad NA, Razak MAA, et al. Prevalence of and factors associated with diarrhoeal diseases among children under five in Malaysia: a cross-sectional study 2016. BMC Public Health. 2018;18(1):1363. Doi:10.1186/s12889-018-6266-z

36. Vélez LF, Sanitato M, Barry D, Alilio M, Apfel F, Coe $G$ et al. The Role of Health Systems and Policy in Producing Behavior and Social Change to Enhance Child Survival and Development in Low- and MiddleIncome Countries: An Examination of the Evidence. J Health Commun. 2014; 19(sup1), 89-121. Doi: http://doi.org/10.1080/10810730.2014.939313

37. Nti CA, Lartey A. Influence of care practices on nutritional status of Ghanaian children. Nutr Res Pract. 2008; 2(2): 93-99. Doi: http://doi.org/10.4162/nrp.2008.2.2.93

38. Vargas J, Molina G. Acceso a los servicios de salud en seis ciudades de Colombia: limitaciones y consecuencias. Rev Fac Nac Salud Pública. 2009; 27(2): 121-30.
39. Finlayson TL, Siefert K, Ismail Al, Sohn W. Maternal self-efficacy and 1-5-year-old children's brushing habits. Community Dent Oral Epidemiol. 2007; 35(4):272-81. Doi: http://doi.org/ 10.1111/j.1600-0528.2007.00313.x

40. Lee S, Kim H, Lee J, Kim J. Association between maternal and child oral health and dental caries in Korea. J Public Health (Berl.) 2019; 27, 219-227. Doi: https://doi.org/10.1007/s10389-018-0936-2

41. Sheiham, A. Oral health, general health and quality of life. Bulletin of the World Health Organization. 2005; 83(9), 644. [internet]. [Consultado 2017 Oct 01]. Disponible en: https://www.ncbi.nlm.nih.gov/pmc/articles/PMC26 26333/ 